International Mathematical Forum, 2, 2007, no. 65, 3199 - 3210

\title{
Equitable Allocation of Shared Costs on Fuzzy Environment
}

\author{
F. Hosseinzadeh Lotfi ${ }^{1}$, G. R. Jahanshahloo, T. Allahviranloo \\ E. Noroozi, A. A. Hosseinzadeh Lotfi \\ Dept. of Math., Science and Research Branch \\ Islamic Azad University, Tehran, Iran
}

\begin{abstract}
In many application to wish data envelopment analysis could be applied there is often a fixed or common cost, wich is imposed on all decision making units $\left(D M U_{s}\right)$.In proposed approach by Cook and kress (1999)of equitable allocation of shared costs between all $D M U_{s}$ several problems should be solved which gives rise to commputational difficulties. G. r. Jahanshahloo et al.presened an approoch in which without solving linear programmin problems only using simple formulas the equitable allocation is achieved. In this paper this approach is imposed on decision making units $(D M U s)$ with fuzzy inputs and outputs in the case that fixed costs are fuzzy.
\end{abstract}

Keywords: Fuzzy linear programming problem, Data Envelopment Analysis(DEA), Fuzzy ranking functions

\section{Introduction}

The use of data envelopment analysis (DEA) for estimating comparative efficiency has become widespread. DEA first introduced by charnes et al.(CCR model) [3] and developed by banker et al.(BBC model)[1] has been widely applied to the efficiency (productivity)measurement of many decisions entities in public and private sectors. In recent years many papers and reports in DEA have indicated the application of this technique in training centers industry etc. One of the most important applications of this technique is allocation of a cost to all decision making units (DMUs). Clearly the cost that is imposed on DMU constitues on additional input which may alter the absolate efficiency rating of the DMU. The objective of mannagement is to allocate those costs in such away that the relative-efficiency is not changed. Hence any allocation

\footnotetext{
${ }^{1}$ Corresponding author. e-mail: hosseinzadeh_lotfi@yahoo.com
} 
that does not alter the relative efficiency measure is equity. Jahanshahloo et al. [5]. proposed on approach for obtaining the equitable cost. Which the fixed cost for one DMU as a new input is added to that DMU in such a way that the relative efficiency is not changed. This method is also performed for DMU with crisp input and output. In this paper the jahanshahloo et al. method is expanded for DMU in which the input and output are triangular fuzzy number and trapezoidal fuzzy number.The Fuzzy mathematical programming in the first category was initially developed by Bellman and Zadeh [2], Tanaka et al. [6] and Zimmermann[7] .This paper is organized in the following way. In section 2 is about back ground, section 3 gives fuzzy DEA introduction. section 4 shows a method for fuzzy equitable allocation of shared costs for all DMU, and a numerical example is solved in section 5 .

\section{Background}

A fuzzy set $\tilde{A}=\left(a_{1}, a_{2}, a_{3}\right)$ is a generalized left right fuzzy numbers of Dubois and Prade[4] if its membership funcation satisfy the following;

$$
\mu_{\tilde{A}}(x)= \begin{cases}L\left(\frac{a_{2}-x}{a_{2}-a_{1}}\right) & , a_{1} \leq x \leq a_{2} \\ 1 & , a_{2} \leq x \leq a_{3} \\ R\left(\frac{x-a_{3}}{a_{4}-a_{3}}\right) & , a_{3} \leq x \leq a_{4} \\ 0 & , \text { otherwise }\end{cases}
$$

where $\mathrm{L}$ and $\mathrm{R}$ are strictly decreasing functions defined on $[0,1]$ and satisfying the conditions

$$
\begin{array}{ll}
L(t)=R(t)=1 & , t \leq 0 \\
L(t)=R(t)=0 & , t \leq 1
\end{array}
$$

for $a_{2}=a_{3}$, we have the classical definition of left right fuzzy numbers (LRFN) of Dubois and Prade[4].Trapezoidal fuzzy numbers (Trfn) are special cases of GLRFN with $\mathrm{L}(\mathrm{t})=\mathrm{R}(\mathrm{t})=1$-t. Triangular Fuzzy Numbers (TFN)are also special cases of General Left and Right Fuzzy Numbers (GLRFN) whit $L(t)=R(t)=1-t$ and $a_{2}=a_{3}$.

\section{3 fuzzy data envelopment analysis}

consider a set of homogeneous DMU as $D M U_{j}, j=1, \ldots, n$.Each DMU consumes $\mathrm{m}$ inputs to produce s outputs. Suppose that $\tilde{x}_{j}=\left(\tilde{x}_{1 j}, \ldots, \tilde{x}_{m j}\right)$ and $\tilde{y}_{j}=\left(\tilde{y}_{1 j}, \ldots, \tilde{y}_{m j}\right)$ are the vectors of input and output values for $D M U_{j}$ respectively. suppose that $\tilde{x}_{i j}$ and $\tilde{y}_{i j}$ are triangular fuzzy numbers (TFN) $\tilde{x}_{i j}=\left(x_{i j}^{l}, x_{i j}^{m}, x_{i j}^{u}\right)$ and $\tilde{y}_{r j}=\left(y_{r j}^{l}, y_{r j}^{m}, y_{r j}^{u}\right)$, 
The primal fuzzy linear programming statement for the(output oriented)CCR model is

$$
\begin{array}{rlr}
\operatorname{Max} & \varphi_{p} & \\
\text { s.t. } & \sum_{j=1}^{n} \lambda_{j} \tilde{x}_{i j} \precsim \tilde{x}_{i p} & , i=1, \ldots, m \\
& \sum_{j=1}^{n} \lambda_{j} \tilde{y}_{r j} \succsim \varphi_{p} \tilde{y}_{r p} & , r=1, \ldots, s \\
& \lambda_{j} \geq 0 & , j=1, \ldots, n
\end{array}
$$

we should note that if $\tilde{A} \precsim \tilde{B}$ if and only if $A^{l} \leq B^{l}, A^{m} \leq B^{m}, \quad A^{u} \leq B^{u}$ [8]. So the model (1) is as follows;

$$
\begin{aligned}
& \operatorname{Max} \varphi_{p} \\
& \text { s.t. } \sum_{j=1}^{n} \lambda_{j} x_{i j}^{l} \leq x_{i p}^{l} \quad, i=1, \ldots, m \\
& \sum_{j=1}^{n} \lambda_{j} x_{i j}^{m} \leq x_{i p}^{m} \quad, i=1, \ldots, m \\
& \sum_{j=1}^{n} \lambda_{j} x_{i j}^{u} \leq x_{i p}^{u} \quad, i=1, \ldots, m \\
& -\sum_{j=1}^{n} \lambda_{j} y_{r j}^{l}+\varphi_{p} y_{r p}^{l} \leq 0 \quad, r=1, \ldots, s \\
& -\sum_{j=1}^{n} \lambda_{j} y_{r j}^{m}+\varphi_{p} y_{r p}^{m} \leq 0 \quad, r=1, \ldots, s \\
& -\sum_{j=1}^{n} \lambda_{j} y_{r j}^{u}+\varphi_{p} y_{r p}^{u} \leq 0 \quad, r=1, \ldots, s \\
& \lambda_{j} \geq 0 \quad, j=1, \ldots, n
\end{aligned}
$$

Also suppose that $\tilde{x_{i j}}$ and $\tilde{y_{r j}}$ are traditional fuzzy numbers $\tilde{x_{i j}}=\left(x_{i j}^{l}, x_{i j}^{m_{1}}, x_{i j}^{m_{2}}, x_{i j}^{u}\right)$ 
and $\tilde{y_{i j}}=\left(y_{i j}^{l}, y_{i j}^{m_{1}}, y_{i j}^{m_{2}}, y_{i j}^{u}\right)$. The model (1)is as follows:

$$
\begin{array}{lll}
\text { Max } & \varphi_{p} & , i=1, \ldots, m \\
\text { s.t. } & \sum_{j=1}^{n} \lambda_{j} x_{i j}^{l} \leq x_{i p}^{l} & , i=1, \ldots, m \\
& \sum_{j=1}^{n} \lambda_{j} x_{i j}^{m_{1}} \leq x_{i p}^{m_{1}} & , i=1, \ldots, m \\
& \sum_{j=1}^{n} \lambda_{j} x_{i j}^{m_{2}} \leq x_{i p}^{m_{2}} & , i=1, \ldots, m \\
& \sum_{j=1}^{n} \lambda_{j} x_{i j}^{u} \leq x_{i p}^{u} & \\
& -\sum_{j=1}^{n} \lambda_{j} y_{r j}^{l}+\varphi_{p} y_{r p}^{l} \leq 0 & , r=1, \ldots, s \\
& -\sum_{j=1}^{n} \lambda_{j} y_{r j}^{m_{1}}+\varphi_{p} y_{r p}^{m_{1}} \leq 0 & , r=1, \ldots, s \\
& -\sum_{j=1}^{n} \lambda_{j} y_{r j}^{m_{2}}+\varphi_{p} y_{r p}^{m_{2}} \leq 0 & , r=1, \ldots, s \\
& -\sum_{j=1}^{n} \lambda_{j} y_{r j}^{u}+\varphi_{p} y_{r p}^{u} \leq 0 & , r=1, \ldots, s \\
\lambda_{j} \geq 0 & , j=1, \ldots, n
\end{array}
$$

$D M U_{p}$ is efficient in the CCR model if and only if $\varphi_{p}^{*}=1$ and in each optimal solution all slacks are equal to zero.

\section{Equitable allocation of shared costs}

We wish to allocate in an cost among n DMUs. Consider $\tilde{R}$ of a fuzzy resource or cost among n DMUs. Consider $\tilde{R}=\left(R^{l}, R^{m}, R^{u}\right)$ and $\tilde{r}_{j}=\left(r_{j}^{l}, r_{j}^{m}, r_{j}^{u}\right) \quad(j=$ $1, \ldots, n)$, so we wish to assign $r_{j}$ to $D M U_{j}(j=1, \ldots, n)$. For determination $r_{j}(j=1, \ldots, n)$ we add it to $D M U_{j}$ as a new input. According to the model 
(1)we have

$$
\begin{array}{lll}
\operatorname{Max} & \varphi_{p} \\
\text { s.t. } & \sum_{j=1}^{n} \lambda_{j} \tilde{x}_{i j} \precsim \tilde{x}_{i p} & , i=1, \ldots, m \\
& \sum_{\substack{n=1 \\
n}}^{n} \lambda_{j} \tilde{y}_{r j} \succsim \varphi_{p} \tilde{y}_{r p} & , r=1, \ldots, s \\
& \sum_{j=1}^{n} \lambda_{j} \tilde{r}_{j} \precsim \tilde{r}_{p} & \\
& \lambda_{j} \geq 0 & , j=1, \ldots, n
\end{array}
$$

noticing to ranking method that defined by Ramik[8] the model (4)is as follows

$$
\begin{array}{lll}
\text { Max } & \varphi_{p}^{\prime} & \\
\text { s.t. } & \sum_{j=1}^{n} \lambda_{j} x_{i j}^{l} \leq x_{i p}^{l} & , i=1, \ldots, m \\
& \sum_{j=1}^{n} \lambda_{j} x_{i j}^{m} \leq x_{i p}^{m} & , i=1, \ldots, m \\
& \sum_{j=1}^{n} \lambda_{j} x_{i j}^{u} \leq x_{i p}^{u} & , i=1, \ldots, m \\
& -\sum_{j=1}^{n} \lambda_{j} y_{r j}^{l}+\varphi_{p}^{\prime} y_{r p}^{l} \leq 0 & , r=1, \ldots, s \\
& -\sum_{j=1}^{n} \lambda_{j} y_{r j}^{m}+\varphi_{p}^{\prime} y_{r p}^{m} \leq 0 & , r=1, \ldots, s \\
& -\sum_{j=1}^{n} \lambda_{j} y_{r j}^{u}+\varphi_{p}^{\prime} y_{r p}^{u} \leq 0 & , r=1, \ldots, s \\
& \sum_{j=1}^{n} \lambda_{j} r_{j}^{l} \leq r_{p}^{l} & \\
\sum_{n=1}^{n} \lambda_{j} r_{j}^{m} \leq r_{p}^{m} & ,(5.1) \\
\sum_{j=1}^{n} \lambda_{j} r_{j}^{u} \leq r_{p}^{u} & \\
\lambda_{j} \geq 0 &
\end{array}
$$

The efficiency value of $D M U_{p}$ by (5) is equal to its value by (2) if and only if the constraint (5-1), (5-2) and (5-3)in (4) be redundant. In other word in order 
to satisfy the invariance condition it is necessary that each optimal solution of (1)be optimal solution of (5). Hence the values of $\left(r_{j}^{l}, r_{j}^{m}, r_{j}^{u}\right)(j=1, \ldots, n)$ have to determine in a manner which the following constraint be redundant

$$
\begin{aligned}
& \sum_{j=1}^{n} \lambda_{j} r_{j}^{l} \leq r_{p}^{l} \\
& \sum_{j=1}^{n} \lambda_{j} r_{j}^{m} \leq r_{p}^{m} \\
& \sum_{j=1}^{n} \lambda_{j} r_{j}^{u} \leq r_{p}^{u}
\end{aligned}
$$

To do so assume that $\lambda^{\prime}$ is an optimal solution of (2)so

$$
\begin{array}{ll}
\sum_{j=1}^{n} \lambda_{j}^{\prime} x_{i j}^{l} \leq x_{i p}^{l} & , i=1, \ldots, m \\
\sum_{j=1}^{n} \lambda_{j}^{\prime} x_{i j}^{m} \leq x_{i p}^{m} & , i=1 \ldots, m \\
\sum_{j=1}^{n} \lambda_{j}^{\prime} x_{i j}^{u} \leq x_{i p}^{u} & , i=1, \ldots, m
\end{array}
$$

By summing the inequalities in (6), (7) and (8)we have:

$$
\begin{aligned}
& \sum_{i=1}^{m}\left(\sum_{j=1}^{n} \lambda_{j}^{\prime} x_{i j}^{l}\right) \leq \sum_{i=1}^{m} x_{i p}^{l} \\
& \sum_{i=1}^{m}\left(\sum_{j=1}^{n} \lambda_{j}^{\prime} x_{i j}^{m}\right) \leq \sum_{i=1}^{m} x_{i p}^{m} \\
& \sum_{i=1}^{m}\left(\sum_{j=1}^{n} \lambda_{j}^{\prime} x_{i j}^{u}\right) \leq \sum_{i=1}^{m} x_{i p}^{u}
\end{aligned}
$$


This ineguality may be written as follows

$$
\begin{aligned}
& \sum_{j=1}^{n} \lambda_{j}^{\prime}\left(\sum_{i=1}^{m} x_{i j}^{l}\right) \leq \sum_{i=1}^{m} x_{i p}^{l} \\
& \sum_{j=1}^{n} \lambda_{j}^{\prime}\left(\sum_{i=1}^{m} x_{i j}^{m}\right) \leq \sum_{i=1}^{m} x_{i p}^{m} \\
& \sum_{j=1}^{n} \lambda_{j}^{\prime}\left(\sum_{i=1}^{m} x_{i j}^{u}\right) \leq \sum_{i=1}^{m} x_{i p}^{u}
\end{aligned}
$$

$$
\text { Suppose } \sum_{j=1}^{n} r_{j}^{l}=R^{l}, \sum_{j=1}^{n} r_{j}^{m}=R^{m}, \sum_{j=1}^{n} r_{j}^{u}=R^{u} \text {. }
$$

By multiplying each side of the inequality (9)by $\frac{R^{l}}{\sum_{q=1}^{n} \sum_{i=1}^{m} x_{i q}}$ By multiplying each side of the inequality(10)by $\frac{R^{m}}{\sum_{q=1}^{n} \sum_{i=1}^{m} x_{i q} m}$ By multiplying each side of the inequality (11)by $\frac{R^{u}}{\sum_{q=1}^{n} \sum_{i=1}^{m} x_{i q} u}$ we have:

$$
\begin{gathered}
\sum_{j=1}^{n} \lambda_{j}^{\prime} \frac{R^{l} \sum_{i=1}^{m} x_{i j}{ }^{l}}{\sum_{q=1}^{n} \sum_{i=1}^{m} x_{i q}{ }^{l}} \leq \frac{R^{l} \sum_{i=1}^{m} x_{i p}{ }^{l}}{\sum_{q=1}^{n} \sum_{i=1}^{m} x_{i q}{ }^{l}} \\
\sum_{j=1}^{n} \lambda_{j}^{\prime} \frac{R^{m} \sum_{i=1}^{m} x_{i j}{ }^{m}}{\sum_{q=1}^{n} \sum_{i=1}^{m} x_{i q}{ }^{m}} \leq \frac{R^{m} \sum_{i=1}^{m} x_{i p}{ }^{m}}{\sum_{q=1}^{n} \sum_{i=1}^{m} x_{i q}{ }^{m}} \\
\sum_{j=1}^{n} \lambda_{j}^{\prime} \frac{R^{u} \sum_{i=1}^{m} x_{i j}{ }^{n}}{\sum_{q=1}^{m} \sum_{i=1}^{m} x_{i q}{ }^{u}} \leq \frac{R^{u} \sum_{i=1}^{m} x_{i p}{ }^{u}}{\sum_{q=1}^{n} \sum_{i=1}^{m} x_{i q}{ }^{u}}
\end{gathered}
$$


so if we choose $r_{j}^{l}, r_{j}^{m}, r_{j}^{u}$ as follows:

$$
\begin{aligned}
r_{j}^{l}= & \frac{R^{l} \sum_{i=1}^{m} x_{i j}{ }^{l}}{\sum_{q=1}^{n} \sum_{i=1}^{m} x_{i q}{ }^{l}}
\end{aligned} \quad, j=1 \ldots, n
$$

the optimal solution for both problems (4) and (5) will be the same from this discussion the following theorem is obvious.

Theorem 4.1. If $\varphi_{p}^{*}$ and $\varphi_{p}^{*}$ are efficiency scores $D M U_{p}$ in (2)and (5)respectively then by choosing $r_{j}$ as relation $(12) \varphi_{p}^{*}=\varphi_{p}^{*}$

Proof: Because constrains (5-1), (5-2) and (5-3)are redundant. So feasible region of model (5) and the model (2) is the same. Therefore optimal solution is the same.

Now we which to allocate in an cost among $\mathrm{n}$ DMUS.consider $\tilde{R}$ of a fuzzy resource or cost among n DMUs. Consider $\tilde{R}=\left(R^{l}, R^{m_{1}}, R^{m_{2}}, R^{u}\right)$ and $\tilde{r}_{j}=$ $\left(r_{j}^{l}, r_{j}^{m_{1}}, r_{j}^{m_{2}}, r_{j}^{u}\right)$

$(j=1, \ldots, n)$ so we wish to assign $r_{j}$ to $D M U_{j}(j=1, \ldots, n)$. For determination $r_{j}$

$(j=1, \ldots, n)$ we add it to $D M U_{j}$ as a new input.According to the model (4)and ranking method that defined by Ramik [8] we have: 


$$
\begin{aligned}
& \operatorname{Max} \varphi_{p}^{\prime \prime} \\
& \text { s.t. } \sum_{j=1}^{n} \lambda_{j} x_{i j}^{l} \leq x_{i p}^{l} \quad, i=1, \ldots, m \\
& \sum_{j=1}^{n} \lambda_{j} x_{i j}^{m_{1}} \leq x_{i p}^{m_{1}} \quad, i=1, \ldots, m \\
& \sum_{j=1}^{n} \lambda_{j} x_{i j}^{m_{2}} \leq x_{i p}^{m_{2}} \quad, i=1, \ldots, m \\
& \sum_{j=1}^{n} \lambda_{j} x_{i j}^{u} \leq x_{i p}^{u} \quad, i=1, \ldots, m \\
& -\sum_{j=1}^{n} \lambda_{j} y_{r j}^{l}+\varphi_{p}^{\prime \prime} y_{r p}^{l} \leq 0 \quad, r=1, \ldots, s \\
& -\sum_{j=1}^{n} \lambda_{j} y_{r j}^{m_{1}}+\varphi_{p}^{”} y_{r p}^{m_{1}} \leq 0 \quad r=1, \ldots, s \\
& -\sum_{j=1}^{n} \lambda_{j} y_{r j}^{m_{2}}+\varphi_{p}^{\prime \prime} y_{r p}^{m_{2}} \leq 0 \quad r=1, \ldots, s \\
& -\sum_{j=1}^{n} \lambda_{j} y_{r j}^{u}+\varphi_{p}^{\prime \prime} y_{r p}^{u} \leq 0 \quad, r=1, \ldots, s \\
& \sum_{j=1}^{n} \lambda_{j} r_{j}^{l} \leq r_{p}^{l} \quad, r=1, \ldots, s \\
& \sum_{j=1}^{n} \lambda_{j} r_{j}^{m_{1}} \leq r_{p}^{m_{1}} \quad, r=1, \ldots, s \\
& \sum_{j=1}^{n} \lambda_{j} r_{j}^{m_{2}} \leq r_{p}^{m_{2}} \quad, r=1, \ldots, s \\
& \begin{array}{ll}
\sum_{j=1}^{n} \lambda_{j} r_{j}^{u} \leq r_{p}^{u} & , r=1, \ldots, s \\
\lambda_{j} \geq 0 & , j=1, \ldots, n
\end{array}
\end{aligned}
$$

there for we consider:

$$
r_{j}^{l}=\frac{R^{l} \sum_{i=1}^{m} x_{i j}{ }^{l}}{\sum_{q=1}^{n} \sum_{i=1}^{m} x_{i q}{ }^{l}} \quad, j=1 \ldots, n
$$




$$
\begin{gathered}
r_{j}^{m_{1}}=\frac{R^{m} \sum_{i=1}^{m} x_{i j}{ }^{m_{1}}}{\sum_{q=1}^{n} \sum_{i=1}^{m} x_{i q}{ }^{m_{1}}} \quad, j=1 \ldots, n \\
r_{j}^{m_{2}}=\frac{R^{m} \sum_{i=1}^{m} x_{i j}{ }^{m_{2}}}{\sum_{q=1}^{n} \sum_{i=1}^{m} x_{i q}{ }^{m_{2}}} \quad, j=1 \ldots, n \\
r_{j}^{u}=\frac{R^{u} \sum_{i=1}^{m} x_{i j}{ }^{u}}{\sum_{q=1}^{n} \sum_{i=1}^{m} x_{i q}{ }^{u}} j=1 \ldots, n
\end{gathered}
$$

the optimal solution for both problems (4)(14)will be the same from this discussion the following theorem is obvious.

Theorem 4.2. If $\varphi_{p}^{*}$ and $\varphi_{p}^{*}$ are efficiency scores $D M U_{p}$ in (3) and (13)respectively then by choosing $r_{j}$ as relation $(14) \varphi_{p}^{*}=\varphi_{p}^{*}$.

\section{$5 \quad$ Examples}

Consider the following table:

Table 1: The fuzzy data

\begin{tabular}{|c|c|c|c|}
\hline$D M U_{j}$ & Input1 & Input2 & Output \\
\hline $\mathrm{A}$ & $(1,3,5)$ & $(1,2,3)$ & $(0.5,1,1.5)$ \\
\hline $\mathrm{B}$ & $(1.5,2,2.5)$ & $(2,25,3)$ & $(2.4,3,3.6)$ \\
\hline $\mathrm{C}$ & $(2.5,3,3.5)$ & $(3.4,4,4.6)$ & $(3,4,5)$ \\
\hline $\mathrm{D}$ & $(4.5,5,5.5)$ & $(1,2,3)$ & $(0.5,1,1.5)$ \\
\hline $\mathrm{E}$ & $(2.5,3,3.5)$ & $(3.4,4,4.4)$ & $(0.5,0.75,1)$ \\
\hline
\end{tabular}

With noticing to model(2), we have:

Table 2: The efficiency

\begin{tabular}{|l|l|l|l|l|}
\hline$\varphi_{A}{ }^{*}=1.2000$ & $\varphi_{B}{ }^{*}=1.0000$ & $\varphi_{C}{ }^{*}=1.0080$ & $\varphi_{D}{ }^{*}=1.2000$ & $\varphi_{E}{ }^{*}=5.0400$ \\
\hline
\end{tabular}

for example $\varphi_{A}{ }^{*}$ can be calculating by using the following model 


$$
\begin{array}{lll}
\operatorname{Max} & \varphi_{p} \\
\text { s.t. } & \lambda_{1}+1.5 \lambda_{2}+2.5 \lambda_{3}+4.5 \lambda_{4}+2.5 \lambda_{5} \leq 1 & \\
& 3 \lambda_{1}+2 \lambda_{2}+3 \lambda_{3}+\lambda_{4}+3 \lambda_{5} \leq 3 & \\
& 5 \lambda_{1}+2.5 \lambda_{2}+3.5 \lambda_{3}+5.5 \lambda_{4}+3.5 \lambda_{5} \leq 5 & \\
& \lambda_{1}+2 \lambda_{2}+3.4 \lambda_{3}+\lambda_{4}+3.4 \lambda_{5} \leq 1 & \\
& 2 \lambda_{1}+2.5 \lambda_{2}+4 \lambda_{3}+2 \lambda_{4}+4 \lambda_{5} \leq 12 & \\
& .5 \lambda_{1}+2.4 \lambda_{2}+3 \lambda_{3}+.5 \lambda_{4}+.5 \lambda_{5} \geq .5 \varphi & \\
& \lambda_{1}+3 \lambda_{2}+4 \lambda_{3}+\operatorname{lambda} a_{4}+.75 \lambda_{5} \geq \varphi & \\
& 1.5 \lambda_{1}+3.6 \lambda_{2}+5 \lambda_{3}+1.5 \lambda_{4}+\lambda_{5} \geq 1.5 \varphi & \\
& \lambda_{j} \geq 0 & j=1, \ldots, n
\end{array}
$$

Now we want to assign the amount $\tilde{R}=(22.8,30.5,38.2)$ to the $D M U$ equitable allocation.So according to model(12)we have:

Table 3: Equitable allocation of shared costs

\begin{tabular}{|c|c|c|c|c|}
\hline$r_{1}^{l}=2$ & $r_{2}^{l}=3.5$ & $r_{3}^{l}=5.9$ & $r_{4}^{l}=5.5$ & $r_{5}^{l}=5.9$ \\
\hline$r_{1}^{m}=5$ & $r_{2}^{m}=5.5$ & $r_{3}^{m}=7$ & $r_{4}^{m}=7$ & $r_{5}^{m}=7$ \\
\hline$r_{1}^{u}=8$ & $r_{2}^{u}=5.5$ & $r_{3}^{u}=8.1$ & $r_{4}^{u}=8.5$ & $r_{5}^{u}=8.1$ \\
\hline
\end{tabular}

If we add three constraints to the model (15)and compute $\varphi_{a}{ }^{*}$ then we see

$\varphi_{a}{ }^{\prime \prime}=\varphi_{a}{ }^{*}=1.2000$.

\section{Conclusion}

Data envelopment analysis technique based on linear programming calculates the relative efficiency of DMUs . This technique has been used in many organizations due to its high efficiency. These applications have developed the theories of the classic models. In most real problems the input and output data (the primary data) are not precise. They are often interval qualitative or ordinal. In this paper, the data are fuzzy. The technique presented here can allocate a fixed cost among the DMUs equitably. In other words, with equitable allocation for all DMUs as a new input, their efficiency doesn't change. This allocation will be fuzzy concerning the primary fuzzy data. In other words, the allocated quantity to all DMUs should be fuzzy. The researchers can develop this technique in a way that the efficiency of the DMUs increases with a particular percentage. This can be done in a fuzzy or absolute environment.

\section{References}

[1] Banker R. D. , A. Charnes, W. W. Cooper, Some models for estimating technical and scale inefficiencies in data envelopment analysis, management Scinecs, 30,9, 1984,1078-1092 . 
[2] Bellman R. E.,L. A. Zadeh,Decision-making in a Fuzzy invironment,Management Sci.17,1970,B141-B164 .

[3] Charnes A., W.W.Cooper and E. Rhodes, Measuring the efficiency of decision making units, European Journal of Operational Research2,6,1978,429444 .

[4] Dubois D., H. Prade ,Systems of linear Fuzzy constraints Fuzzy sets and systems 3,1980,37-48 .

[5] Jahanshahloo G. R.,F. Hosseienzadeh Lotfi, N. Shoja and M. Sanei, An alternative approach for equitable allocation of shared costs by using DEA, Applied Mathematics and computation, 153,2004,267-274 .

[6] Tanaka H.,T. Okuda, K. Asai , On Fuzzy mathematical programming, J.Cybernet,3, $1974,37-46$.

[7] Zimmermann H.G., Description and optimization of fuzzy system, Internat. J. general system, 2,1967,209-215 .

[8] Ramik J., J. Rimanek, In equlity relation between fuzzy sets number and its use in fuzzy optimization, Fuzzy Sets an Systems, 16 ,1985,123-138 .

Received: September 5, 2007 